\title{
From inflation to a zero cosmological constant phase without fine tuning
}

\author{
E. I. Guendelman* and A. B. Kaganovich ${ }^{\dagger}$ \\ Physics Department, Ben Gurion University of the Negev, Beer Sheva 84105, Israel
}

\begin{abstract}
We show that it is possible to obtain inflation and also solve the cosmological constant problem. The theory is invariant under changes of the Lagrangian density $L$ to $L+$ const. Then the constant part of a scalar field potential $V$ cannot be responsible for inflation. However, we show that inflation can be driven by a condensate of a four index field strength. A constraint appears which correlates this condensate to $V$. After a conformal transformation, the equations are the standard GR equations with an effective scalar field potential $V_{\text {eff }}$ which has generally an absolute minimum $V_{e f f}=0$ independently of $V$ and without fine tuning. We also show that, after inflation, the usual reheating phase scenario (from oscillations around the absolute minimum) is possible.
\end{abstract}

One of the biggest puzzles of modern physics is what is referred to as the "cosmological constant problem", i.e. the absence of a possible constant part of the vacuum energy in the present day universe [1]. On the other hand, many questions in modern cosmology appear to be solved by the so called "inflationary model" which makes use of a big effective cosmological constant in the early universe [2]. A possible conflict between a successful resolution of the cosmological constant problem and the existence of an inflationary phase could be a "potential Achilles heel for the scenario" as has been pointed out [3]. Here we will see that indeed there is no conflict between the existence of an inflationary phase and the disappearance of the cosmological constant in the later phases of cosmological evolution (without the need of fine tuning).

In Refs. [4], [5], [6] we have developed an approach where the cosmological constant problem is treated as the absence of gravitational effects of a possible constant part of the Lagrangian density. In order to achieve this, we assume that the measure of integration in the action is not necessarily $\sqrt{-g} \quad\left(g=\operatorname{Det}\left(g_{\mu \nu}\right)\right)$ but it is determined dynamically through additional degrees of freedom. This theory is based on the demand that such measure respects the principle

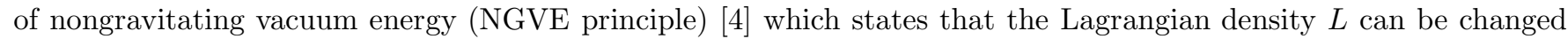
to $L+$ constant without affecting the dynamics. This requirement is imposed in order to offer a new approach for the solution of the cosmological constant problem. Clearly this invariance is achieved if the measure of integration in the action is a total derivative. This is satisfied if the measure appropriate to the integration in the space of 4 scalar fields $\varphi_{a}, \quad a=1, \ldots, 4$, that is $d V=d \varphi_{1} \wedge \ldots \wedge d \varphi_{4} \equiv \frac{\Phi}{4 !} d^{4} x$ where $\Phi \equiv \varepsilon_{a_{1} \ldots a_{4}} \varepsilon^{\mu_{1} \ldots \mu_{4}}\left(\partial_{\mu_{1}} \varphi_{a_{1}}\right) \ldots\left(\partial_{\mu_{4}} \varphi_{a_{4}}\right)$.

There are two well known variational principles: the first and the second order formalisms, which are equivalent in the case of the general theory of relativity (GR). However, as it was shown [5], the first and the second order formalisms (starting from the same looking form of Lagrangian) are not equivalent in the context of the NGVE theories. The NGVE theory in the first order formalism leads to the resolution of the cosmological constant problem in a straightforward way [5], [6] and in this paper, we will follow this approach. Furthemore, in the spirit of the theory, which assumes independence of the measure degrees of freedom from $g_{\mu \nu}$, the first order formalism where the independence of $\Gamma_{\mu \nu}^{\lambda}$ and $g_{\mu \nu}$ in the action is assumed, is of course much more natural.

According to the NGVE-principle, the total action in the 4-dimensional space-time should be written in the form $S=\int \Phi L d^{4} x$. We assume that $L$ does not contain the measure fields $\varphi_{a}$, that is the fields by means of which

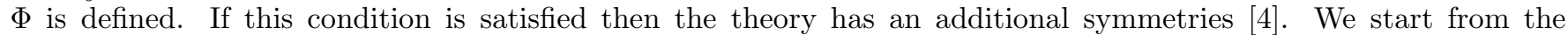
total Lagrangian density $L=\kappa^{-1} R(\Gamma, g)+L_{m}$, where $L_{m}$ is the matter Lagrangian density and $R(\Gamma, g)$ is the scalar curvature $R(\Gamma, g)=g^{\mu \nu} R_{\mu \nu}(\Gamma)$ of the space-time with the affine connection $\Gamma_{\mu \nu}^{\lambda}$ (the first order formalism is used,that is in the action, the connection $\Gamma_{\mu \nu}^{\lambda}$ and the metric $g_{\mu \nu}$ are dynamically independent variables), $R_{\mu \nu}(\Gamma)=R_{\mu \nu \alpha}^{\alpha}(\Gamma)$, $R_{\mu \nu \beta}^{\alpha}(\Gamma) \equiv \Gamma_{\mu \nu, \beta}^{\alpha}+\Gamma_{\lambda \beta}^{\alpha} \Gamma_{\mu \nu}^{\lambda}-(\nu \leftrightarrow \beta)$. This curvature tensor is invariant under the $\lambda$ - transformation [7] $\Gamma_{\mu \nu}^{\prime \alpha}=$ $\Gamma_{\mu \nu}^{\alpha}+\delta_{\mu}^{\alpha} \lambda,_{\nu}$. Its importance in the NGVE-theory is that allows us to eliminate the contribution to the torsion which appears as a result of introduction of the new measure [6]. However, even after we fix a gauge where this contribution to the torsion disappears, still there is the non metric contribution [6] to the symmetric connection related to the measure. In fact, solving the equations following from the variation of the action with respect to $\Gamma_{\mu \nu}^{\lambda}$ (in the case where $L_{m}$ does not depend on the connection $\Gamma_{\mu \nu}^{\lambda}$ ) and making use the appropriate $\lambda$ - transformation, we get

$$
\Gamma_{\mu \nu}^{\lambda}=\left\{\begin{array}{l}
\lambda \\
\mu \nu
\end{array}\right\}+\Sigma_{\mu \nu}^{\lambda}
$$

*GUENDEL@BGUmail.BGU.AC.IL

${ }^{\dagger}$ ALEXK@BGUmail.BGU.AC.IL 
where $\left\{\begin{array}{c}\alpha \\ \mu \nu\end{array}\right\}$ are the Christoffel's connection coefficients and

$$
\begin{aligned}
\Sigma_{\mu \nu}^{\lambda}(\sigma) & =\frac{1}{2}\left(\delta_{\mu}^{\lambda} \sigma,_{\nu}+\delta_{\nu}^{\lambda} \sigma,{ }_{\mu}-\sigma,{ }_{\alpha} g_{\mu \nu} g^{\lambda \alpha}\right) \\
\sigma & \equiv \ln \chi, \quad \chi \equiv \Phi / \sqrt{-g} .
\end{aligned}
$$

In addition to this, in the vacuum and in some matter models, the theory possesses a local symmetry which plays a major role. This symmetry consists of a conformal transformation of the metric $g_{\mu \nu}^{\prime}(x)=J(x) g_{\mu \nu}(x)$ accompanied by a corresponding diffeomorphism $\varphi_{a} \rightarrow \varphi_{a}^{\prime}=\varphi_{a}^{\prime}\left(\varphi_{b}\right)$ in the space of the scalar fields $\varphi_{a}$ such that $J=\operatorname{Det}\left(\frac{\partial \varphi_{a}^{\prime}}{\partial \varphi_{h}}\right)$. Then for $\Phi$ we have: $\Phi^{\prime}(x)=J(x) \Phi(x)$. In the presence of fermions this symmetry is appropriately generalized [6]. For models where it holds it is possible to choose a gauge where the measure $\Phi$ coincides with the measure of general relativity $\sqrt{-g}$. This is why we call this symmetry "local Einstein symmetry" (LES).

Varying the action with respect to $\varphi_{a}$ we get $A_{b}^{\alpha} \partial_{\alpha}\left[-\frac{1}{\kappa} R(\Gamma, g)+L_{m}\right]=0$ where $A_{b}^{\alpha}=$ $\varepsilon_{a_{1} a_{2} a_{3} b} \varepsilon^{\mu_{1} \mu_{2} \mu_{3} \alpha}\left(\partial_{\mu_{1}} \varphi_{a_{1}}\right)\left(\partial_{\mu_{2}} \varphi_{a_{2}}\right)\left(\partial_{\mu_{3}} \varphi_{a_{3}}\right)$. Since $A_{b}^{\alpha} \partial_{\alpha} \varphi_{b^{\prime}}=\frac{1}{4} \delta_{b b^{\prime}} \Phi$ it follows that $\operatorname{Det}\left(A_{b}^{\alpha}\right)=\frac{4^{-4}}{4 !} \Phi^{3}$. If $\Phi \neq 0$, we obtain

$$
-\frac{1}{\kappa} R(\Gamma, g)+L_{m}=M=\text { constant } .
$$

Varying the action with respect to $g^{\mu \nu}$ we get (for simplicity we present here the calculations for the case when there are no fermions)

$$
-\frac{1}{\kappa} R_{\mu \nu}(\Gamma)+\frac{\partial L_{m}}{\partial g^{\mu \nu}}=0
$$

In the case where $L_{m}$ does not depend on $\Gamma_{\mu \nu}^{\alpha}$, Eq. (何) takes the form

$$
\square \chi^{1 / 2}-\frac{1}{6}\left[R(g)-\kappa\left(L_{m}-M\right)\right] \chi^{1 / 2}=0
$$

where $R(g)$ is the Riemannian scalar curvature.

Contracting Eq. (5) with $g^{\mu \nu}$ and making use Eq. (4) we get the constraint

$$
g^{\mu \nu} \frac{\partial\left(L_{m}-M\right)}{\partial g^{\mu \nu}}-\left(L_{m}-M\right)=0 .
$$

For the cases where the LES is an exact symmetry, we can eliminate the mentioned above $\chi$-contribution to the connection. Indeed, for $J=\chi$ we get $\chi^{\prime} \equiv 1$ and $\Gamma_{\mu \nu}^{\alpha \alpha}=\left\{\begin{array}{c}\alpha \\ \mu \nu\end{array}\right\}^{\prime}$, where $\left\{\begin{array}{l}\alpha \\ \mu \nu\end{array}\right\}^{\prime}$ are the Christoffel's coefficients corresponding to the new metric $g_{\mu \nu}^{\prime}$. In this gauge the metric-affine space-time becomes a Riemannian space-time (in the absence of fermions).

In the presence of matter, the LES may be lost. However, the theory still makes sense [5] and the resolution of the cosmological constant problem is retained. Together with this, the LES appears to have a deep geometric meaning [6].

Now let us consider cases when the constraint (7) is not satisfied without restrictions on the dynamics of the matter fields. Nevertheless, the constraint (7) holds as a consequence of the variational principle in any situation.

A simple situation where the constraint (7) is not automatic is the case of a single scalar field with a nontrivial potential $V(\phi)$. In this case the constraint (6) implies

$$
V(\phi)+M=0 .
$$

Therefore we conclude that, provided $\Phi \neq 0$, there is no dynamics for the theory of a single scalar field, since the constraint (8) forces this scalar field to be a constant [5]. Inserting (8) into (6), we obtain $\square \chi^{1 / 2}-\frac{1}{6} R(g) \chi^{1 / 2}=0$. This conformal coupling of $\chi^{1 / 2}$ with $R(g)$ shows that on the mass shell the LES is restored (this can be seen in all the other equations as well). Making use then of the gauge $\chi=1$, we see that $R(g)=0$ and therefore the only maximally symmetric solution is Minkowski space. Consistency with the scalar field equation requires $V^{\prime} \equiv \frac{\partial V}{\partial \phi}=0$, that is $\phi$ is located on an extremum of $V(\phi)$.

As it follows from our analysis above, a model with only a scalar field cannot give rise to inflation since the gravitational effects of the scalar field potential is always cancelled by the integration constant $M$. We will see that 
nontrivial dynamics of a single scalar field including the possibility of inflation can be obtained by considering a model with an additional degree of freedom described by a three-index potential $A_{\beta \mu \nu}$ as in the Lagrangian density

$$
L=-\frac{1}{\kappa} R(\Gamma, g)+\frac{1}{2} \phi, \alpha{ }^{, \alpha}-V(\phi)+\frac{1}{4 !} F_{\alpha \beta \mu \nu} F^{\alpha \beta \mu \nu} .
$$

Here $F_{\alpha \beta \mu \nu} \equiv \partial_{[\alpha} A_{\beta \mu \nu]}$ is the field strength which is invariant under the gauge transformation $A_{\beta \mu \nu} \rightarrow A_{\beta \mu \nu}+\partial_{[\beta} f_{\mu \nu]}$. In ordinary 4-dimensional GR, the $F_{\alpha \beta \mu \nu} F^{\alpha \beta \mu \nu}$ term gives rise to a cosmological constant depending on an integration constant [8]. In our case, due to the constraint (7), the degrees of freedom contained in $F_{\alpha \beta \mu \nu}$ and those of the scalar field $\phi$ will be intimately correlated. The sign in front of the $F_{\alpha \beta \mu \nu} F^{\alpha \beta \mu \nu}$ term is chosen so that in this model the resulting expression for the energy density of the scalar field $\phi$ is a positive definite one for any possible space-time dependence of $\phi$ in an effective "Einstein picture" (see below). Notice also that two last terms in the action with the Lagrangian (9) break explicitly the LES.

The gravitational equations (5) take now the form

$$
-\frac{1}{\kappa} R_{\mu \nu}(\Gamma)+\frac{1}{2} \phi, \mu \phi, \nu+\frac{1}{6} F_{\mu \alpha \beta \gamma} F_{\nu}^{\alpha \beta \gamma}=0 .
$$

Notice that the scalar field potential $V(\phi)$ does not appear explicitly in Eqs. (10). However, the constraint (7), which takes now the form

$$
V(\phi)+M=-\frac{1}{8} F_{\alpha \beta \mu \nu} F^{\alpha \beta \mu \nu},
$$

allows us to express the last term in (10) in terms of the potential $V(\phi)$ (using the fact that $F^{\alpha \beta \mu \nu} \propto \varepsilon^{\alpha \beta \mu \nu}$ in 4-dimensional space-time).

Varying the action with respect to $A_{\nu \alpha \beta}$, we get the equation $\partial_{\mu}\left(\Phi F^{\mu \nu \alpha \beta}\right)=0$. Its general solution has a form (see definition (3)).

$$
F^{\alpha \beta \mu \nu}=\frac{\lambda}{\Phi} \varepsilon^{\alpha \beta \mu \nu} \equiv \frac{\lambda}{\chi \sqrt{-g}} \varepsilon^{\alpha \beta \mu \nu},
$$

where $\lambda$ is an integration constant. Then $F_{\alpha \beta \mu \nu} F^{\alpha \beta \mu \nu}=-\lambda^{2} 4 ! / \chi^{2}$ and therefore

$$
V(\phi)+M=3 \lambda^{2} / \chi^{2}
$$

and $F_{\mu \alpha \beta \gamma} F_{\nu}^{\alpha \beta \gamma}=-\left(6 \lambda^{2} / \chi^{2}\right) g_{\mu \nu}=-2[V(\phi)+M] g_{\mu \nu}$ (notice that $F_{\alpha \beta \mu \nu} F^{\alpha \beta \mu \nu}$ is not a constant now as opposed to the GR case (8)). This shows how the potential $V(\phi)$ appears in Eq. (10), spontaneously violating the symmetry of the action $V(\phi) \rightarrow V(\phi)+$ constant, which now corresponds to a redefinition of the integration constant $M$.

The equation of motion of the scalar field $\phi$ is $(-g)^{-1 / 2} \partial_{\mu}\left(\sqrt{-g} g^{\mu \nu} \partial_{\nu} \phi\right)+\sigma,_{\mu} \phi^{\mu}+V^{\prime}(\phi)=0$, where $V^{\prime} \equiv \frac{\partial V}{\partial \phi}$.

The derivatives of the field $\sigma$ enter both in the gravitational Eqs. (10) (through the connection) and in the scalar field equation. In order to see easily the physical content of this model, we have to perform a conformal transformation $\bar{g}_{\mu \nu}(x)=\chi g_{\mu \nu}(x)$ to obtain an "Einstein picture". Notice that now this transformation is not a symmetry and indeed changes the form of equations. In this new frame, the gravitational equations become those of GR in the Riemannian space-time with metric $\bar{g}_{\mu \nu}$

$$
R_{\mu \nu}\left(\bar{g}_{\alpha \beta}\right)-\frac{1}{2} \bar{g}_{\mu \nu} R\left(\bar{g}_{\alpha \beta}\right)=\frac{\kappa}{2} T_{\mu \nu}^{e f f}(\phi),
$$

where the source is the minimally coupled scalar field $\phi$

$$
T_{\mu \nu}^{e f f}(\phi)=\phi_{, \mu} \phi_{, \nu}-\frac{1}{2} \bar{g}_{\mu \nu} \phi_{\alpha} \phi_{\beta} \bar{g}^{\alpha \beta}+\bar{g}_{\mu \nu} V_{e f f}(\phi)
$$

with the new effective potential

$$
V_{e f f} \equiv \frac{2}{\lambda 3 \sqrt{3}}(V+M)^{3 / 2}
$$

The scalar field equation takes a conventional form $(-\bar{g})^{-1 / 2} \partial_{\mu}\left(\sqrt{-\bar{g}} \bar{g}^{\mu \nu} \partial_{\nu} \phi\right)+V_{e f f}^{\prime}(\phi)=0$. Notice again that the potential $V_{e f f}(\phi)$ is non negative one which is a result of the choice of sign in front of the $F_{\alpha \beta \mu \nu} F^{\alpha \beta \mu \nu}$ term in (9)). 
We see that in the Einstein picture, for any analytic $V(\phi), V_{e f f}(\phi)$ has an extremum, that is $V_{e f f}^{\prime}=0$, either when $V^{\prime}=0$ or $V+M=0$. The extremum when $V+M=0$ corresponds to an absolute minimum (since $V_{\text {eff }}(\phi)$ is non negative) and therefore it is a vacuum with zero effective cosmological constant. It should be emphasized that all what is required is that $V+M$ touches zero at some point $\phi_{0}$ but $V^{\prime}$ at this point does not need to vanish. Therefore no fine tuning in the usual sense, of adjusting a minimum of a potential to coincide with the point where this potential itself vanishes, is required. And the situation is even more favorable since even if $V+M$ happens not to touch zero for any value of $\phi$, we always have an infinite set of other integration constants where this will happen.

In the context of the cosmology, for the Friedmann- Robertson-Walker universe where in the Einstein picture $\phi=\phi(t)$ and $d \bar{s}^{2}=\bar{g}_{\mu \nu} d x^{\mu} d x^{\nu}=d t^{2}-\bar{a}^{2}(t) d l^{2}, d l^{2}=\left[d r^{2} /\left(1-k r^{2}\right)+r^{2} d \Omega^{2}\right]$, we notice that due to the positivity of $V_{e f f}$, all the known inflationary scenarios [2] for the very early universe can be implemented depending on the choice of the potential $V(\phi)$. It is very interesting that the parameters ruling the inflation are controlled by the integration constants $M$ and $\lambda$.

After inflation, when the scalar field $\phi$ approaches the position $\phi_{0}$ of the absolute minimum of the potential $V_{\text {eff }}$, the $\chi$-field approaches infinity as it seen from the constraint (13). To clarify the meaning of this effect, let us go back to the picture with the original $g_{\mu \nu}$ while still using the cosmic time $t$ that was defined in the Einstein picture. Then equation for $\phi$ is

$$
\ddot{\phi}+3 \frac{\dot{a}}{a} \dot{\phi}-\frac{3 V^{\prime}}{4(V+M)} \dot{\phi}^{2}+\frac{\sqrt{V+M}}{\lambda \sqrt{3}} V^{\prime}=0,
$$

where $a^{2}(t)=\bar{a}^{2}(t) / \chi(t), g_{00}(t)=1 / \chi(t)$ and constraint (13) have been used.

Generally, $\dot{\phi}$ does not go to zero as $\phi \rightarrow \phi_{0}$ (and $V(\phi)+M \rightarrow 0$ ). In this asymptotical region we can find the first integral of Eq. (17). Assuming that $V^{\prime}\left(\phi_{0}\right) \neq 0$, i.e. without fine tuning, we get

$$
\dot{\phi} a^{3}(t) \simeq c[V(\phi)+M]^{3 / 4}, \quad c=\text { const } \quad\left(\text { as } \quad \phi \rightarrow \phi_{0}\right),
$$

which means that $a(t) \rightarrow 0$ as $\phi \rightarrow \phi_{0}$ (notice that if we would have chosen a coordinate frame in the original picture such that $d s^{2}=d t^{\prime 2}-a^{2}\left(t^{\prime}\right) d l^{2}$, then instead of (18) we would have gotten $a^{3}\left(t^{\prime}\right) d \phi / d t^{\prime} \simeq c[V(\phi)+M]^{1 / 2}$ as $\left.\phi \rightarrow \phi_{0}\right)$. Then integrating the gravitational equations we get asymptotically (as $\phi \rightarrow \phi_{0}$ ) that $a^{2}(t)=a_{0}^{2} / \chi(t), \quad a_{0}=$ const, that is in the original frame there is a collapse of the universe from a finite $a$ to $a=0$ in a finite time and therefore the Riemannian curvature goes to infinity as $\phi \rightarrow \phi_{0}$. This pathology is not seen in the Einstein frame due to the singularity of the conformal transformation $\bar{a}^{2}=\chi a^{2}$ at $\phi=\phi_{0}$. In fact, this is not a problem from the point of view of physics, since as $\phi \rightarrow \phi_{0}$ (and $V(\phi)+M \rightarrow 0$ ), the LES becomes restored at the critical point $\phi \equiv \phi_{0}$ where $V\left(\phi_{0}\right)+M=0$. In the presence of the LES, the conformal transformation $\bar{g}_{\mu \nu}(x)=\chi g_{\mu \nu}(x)$ becomes part of the LES transformation and represents a nonsingular gauge choice.

A real problem in the scenario discussed above is the fact that at the point $\phi=\phi_{0}$ we have $V_{\text {eff }}=0, V_{e f f}^{\prime}=0$ but $V_{e f f}^{\prime \prime}$ diverges at $\phi=\phi_{0}$. This causes problems both in the cosmological picture when considering the possibility of small oscillations arround the minimum and in the associated particle physics, since masses of scalars, like for example the Higgs mass, will appear always infinite.

It turns out that the model with the Lagrangian density (9) described above is only a representative of a family of possible models with actions $S=\int \Phi L d^{4} x$ where

$$
L=-\frac{1}{\kappa} R(\Gamma, g)+\frac{1}{2} \phi,_{\alpha} \phi^{, \alpha}-V(\phi)-\frac{1}{4 p-1}\left(-F_{\alpha \beta \mu \nu} F^{\alpha \beta \mu \nu}\right)^{p},
$$

where still $F_{\alpha \beta \mu \nu} \equiv \partial_{[\alpha} A_{\beta \mu \nu]}$. Here $p \neq 1 / 4$ is a real number parametrizing the family of Lagrangians (19). In this case, once again solving the equation of motion obtained from variation with respect to $A_{\mu \nu \alpha}$ and then using the associated constraint that replaces (11) we obtain instead of Eq.(13)

$$
\lambda^{2} / \chi^{2}=\frac{1}{24}(V(\phi)+M)^{2-1 / p}
$$

Then instead of Eq. 16 the associated effective potential in the Einstein picture is

$$
V_{\text {eff }} \equiv \frac{1}{\lambda \sqrt{24}}(V+M)^{2-1 / 2 p} .
$$

As before, the extremum when $V+M=0$ corresponds to an absolute minimum (for any $p>1 / 2$ ) and therefore it is a vacuum with zero effective cosmological constant. We can now notice that the limit $p \rightarrow \infty$ is critical one, since in 
this limit $V_{e f f}=\frac{1}{\lambda \sqrt{24}}(V+M)^{2}$ and for any analytical function $V(\phi)$, all derivatives of the effective potential $V_{\text {eff }}(\phi)$ are finite at the absolute minimum $\phi=\phi_{0}$ where $V\left(\phi_{0}\right)+M=0$. In particular, $V_{e f f}^{\prime \prime}\left(\phi_{0}\right) \propto\left[V^{\prime}\left(\phi_{0}\right)\right]^{2}$ is finite (and nonzero if we do not carry out the fine tuning $\left.V^{\prime}\left(\phi_{0}\right)=0\right)$. Therefore the Higgs boson, in particular, can reappear as a physical particle of the theory. In the context of cosmology where $V_{\text {eff }}(\phi)$ plays the role of the inflaton potential, a finite mass of the inflaton allows to recover the usual oscillatory regime of the reheating period after inflation that are usually considered.

The incorporation of gauge fields and fermions into the family of Lagrangians (19) has also many interesting features in the Einstein picture like the appearence of normal Maxwell dynamics in the low energy limit in the abelian case and standard Yang-Mills behavior in the nonabelian case, the appearence of mass for fermions, etc.. In the associated particle physics, the $p \rightarrow \infty$ limit has remarkable features as well. These subjects will be studied in a longer publication [9].

As one of the referees of this paper pointed out, the picture presented here should be regarded as a preliminary one. In particular, questions that concern reheating and density perturbations have to be analyzed. Here, in addition to the usual possibility of choosing the potential of the scalar field which governs the cosmological processes mentioned before, this model has the additional integration constants $M$ and $\lambda$ which also enter in the effective potential. Therefore we hope that this model will provide more possibilities to obtain naturally the correct density perturbations and reheating.

[1] S. Weinberg, Rev. Mod. Phys. 61, 1 (1989); Y. J. Ng, Int. J. Mod. Phys. D1, 145, (1992); Gravitation and Modern Cosmology, The Cosmological Constant Problem, edited by A. Zichichi, V. de Sabbata and N. Sanchez (Ettore Majorana International Science Series, Plenum Press, 1991). For the history of the problem see for example discussion in I. Novikov, Evolution of the Universe, Cambridge University Press, 1983.

[2] A. H. Guth, Phys. Rev. D23, 347 (1981); A. D. Linde, Phys. Lett. 108B, 389 (1982); A. Albrecht and P. J. Steinhardt, Phys. Rev. Lett. 48, 1220 (1982), A. A. Starobinsky, Phys. Lett. B91, 99 (1980); A. D. Linde, Phys. Lett. B129, 177 (1983); D. La and D. J. Steinhardt, Phys. Rev. Lett. 62, 376 (1989).

[3] See, for example: E. Kolb and M. S. Turner, The Early Universe, Addison Wesley, 1990 (see p.314).

[4] E.I. Guendelman and A.B. Kaganovich, Phys. Rev. D53, 7020 (1996).

[5] E.I. Guendelman and A.B. Kaganovich, Phys. Rev.D55, 5970 (1997).

[6] E.I. Guendelman and A.B. Kaganovich, Phys. Rev.D56 (1997).

[7] A.Einstein, The Meaning of Relativity, Fifth Edition, MJF Books, N.Y.1956 ( see Appendix II).

[8] A. Aurilia, H. Nicolai and P. K. Townsend, Nucl.Phys. B176, 509 (1980); S. W. Hawking, Phys. Lett., 134B, 276 (1984); A. Aurilia, G. Denardo, F. Legovini and E. Spalluci, ibid., 147B, 258 (1984); E. Witten , in Shelter II 1985 Proc. 1983 Shelter Island Conference on Quantum Field Theory and the Fundamental Problems of Physics (Cambridge, MA: MIT Press); J. D. Brown and C. Teitelboim, Phys. Lett. 195B, 177 (1987); Nucl. Phys. B297, 787 (1988).

[9] E.I. Guendelman and A.B. Kaganovich, in preparation. 